Ann. Génét. Sél. anim., I977, 9 (2), 24I-245.

\title{
Relations entre la croissance et l'activité en open-field chez la Poule \\ I. -- Effet de la sélection pour l'activité en open-field sur la croissance en poids et la conformation
}

\author{
J. M. FAURE
}

avec la collaboration technique de F. Morozeau et G. Marche

Station expérimentale d'Aviculture du Magneraud, I.N.R.A., B.P. 52, I7700 Surgères (France)

\begin{abstract}
Résumé
Nous avons comparé la croissance de deux souches sélectionnées pour leur activité en openfield (mesure d'émotivité). On constate que les animaux les plus émotifs (inactifs) ont la meilleure croissance aussi bien au point de vue du poids que de la croissance des os longs. Ces données sont en contradiction avec les données bibliographiques montrant que les animaux les plus sensibles aux stress ou les plus stressés ont leur croissance diminuée.

Cette contradiction est expliquée en supposant qu'il y a eu une dérive génétique due aux faibles effectifs.
\end{abstract}

\section{Introduction}

Les relations entre phénomènes de stress et croissance peuvent être étudiées par deux méthodes. On peut soit faire varier des facteurs stressants du milieu, en général la densité (Scholtysse K, I973; Manoharan et Michael, I974; Ebedes, I974), soit comparer des souches différentes par leur sensibilité aux stress (BROWN et NESTOR, I973, I974 $a$ et $b$ ). Dans la plupart des cas, on constate que les animaux les plus sensibles aux stress ou placés dans les conditions les plus stressantes ont la croissance la plus faible.

Nous avons voulu utiliser les deux aspects possibles de l'approche génétique, en étudiant d'une part l'influence d'une sélection pour l'émotivité sur la croissance et d'autre part l'influence d'une sélection pour la croissance sur l'émotivité.

Dans ce premier travail, nous avons essayé d'étudier cette relation entre 
stress et croissance sur deux souches de poulets sélectionnées de manière divergente pour leur activité en open-field (FAURE, FOLMER, 1975). Ces souches diffèrent à la fois pour leur émotivité mesurée par des tests éthologiques (FAURE, I975) et pour leur taux de corticostérone (ROMBAUTS, FAURE, à paraître). Nous avons comparé la croissance de ces deux souches dans deux milieux différents : sol et cage.

\section{Matériel et méthodes}

A partir d'une souche Cornish de type commercial, deux lignées ont été sélectionnées de manière divergente pour leur activité en open-field, à l'âge de deux jours (une souche active $\mathrm{A}$ et une souche inactive I).

A chaque génération, environ Ioo animaux par sexe et par souche sont élevés sexes et souches séparés. L'élevage se fait au sol dans 4 cases de poussinière jusqu'à I2 semaines, puis en poulailler dans 4 cases de $32 \mathrm{~m}^{2}$. Les animaux sont nourris ad libitum. Tous les animaux sont pesés à 6 , I4 et 20 semaines d'âge. En plus de la pesée à 6 semaines, sont mesurés l'angle de poitrine, la longueur du bréchet, la longueur et le diamètre du tarse.

De plus, à la $2^{\mathrm{e}}$ et à la $4^{\mathrm{e}}$ génération, environ $5^{\circ}$ animaux par sexe et par souche ont été élevés en cage d'engraissement par groupes d'environ I 2 animaux par cage de $\mathrm{I}^{2}$ de surface. Ces animaux ont été pesés à l'âge de 7 semaines.

Toutes les comparaisons entre les deux souches ont été faites par un test $t$ de Student.

\section{Résultats}

Les résultats des pesées sont représentés dans le tableau I.

On peut constater pour les mâles que des différences significatives de poids à 6 semaines n'existent, pour les deux souches, que pour la $3^{\mathrm{e}}$ génération mais qu'il y a des différences significatives pour le poids à I4 semaines aux $3^{\mathrm{e}}$ et $4^{\mathrm{e}}$ générations et pour le poids à 20 semaines dès la $\mathrm{I}^{\mathrm{re}}$.

Pour les poules, les différences sont significatives dès la Ire génération et pour tous les âges à une exception près (20 semaines, $3^{\mathrm{e}}$ génération).

Les résultats de croissance des animaux élevés en cage sont indiqués dans le tableau 2. Aucune différence n'est significative.

Les résultats des mesures de conformation sont présentés dans le tableau 3. On constate que pour toutes les mesures, excepté celle de la génération $O$, les valeurs moyennes des inactifs sont toujours supérieures à celles des actifs et presque toujours significatives, au moins aux $\mathrm{F}_{3}$ et $\mathrm{F}_{4}$.

\section{Discussion}

Nous constatons donc que la sélection pour l'activité en open-field a entraîné, pour la souche inactive, une augmentation du poids mais aussi de l'importance des muscles pectoraux (angle de poitrine), de la croissance en longueur (longueur du 
TABLEAU I

Evolution du poids des animaux provenant de souches sélectionnées en open-field élevées au sol. Evolution of weight of animals from lines selected in open field, on floor

\begin{tabular}{|c|c|c|c|c|c|c|c|}
\hline & & \multicolumn{2}{|c|}{ Poids 6 semaines } & \multicolumn{2}{|c|}{ Poids I 4 semaines } & \multicolumn{2}{|c|}{ Poids 20 semaines } \\
\hline & $\begin{array}{l}\text { Génération } \\
\text { de sélect. }\end{array}$ & A & I & A & I & A & 1 \\
\hline \multirow{5}{*}{$\sigma^{*}$} & $F_{0}$ & I $34^{\mathrm{r}}$ & I 380 & 33 I 8 & 3213 & 4273 & $42 \mathrm{II}$ \\
\hline & $\mathrm{F}_{1}$ & I 252 & I 249 & 3090 & 3125 & 3938 & $4223(* * *)$ \\
\hline & $\quad \mathbf{F}_{\mathbf{2}}$ & $\begin{array}{r}1279 \\
\end{array}$ & 1310 & 3220 & $334^{\circ}$ & 3892 & $4216(* * *)$ \\
\hline & $\mathrm{F}_{3}$ & I 4 I I & I $500(* * *)$ & 3366 & $3668(* * *)$ & 4243 & $4762(* * *)$ \\
\hline & $\mathrm{F}_{4}$ & I 68 & I $\operatorname{I}^{7} 8$ & 3329 & $3473(* *)$ & $4 \mathrm{I} 82$ & $43^{87}\left(^{*}\right)$ \\
\hline \multirow{5}{*}{ 옹 } & $F_{0}$ & I 226 & I 208 & 2679 & 2628 & 3772 & $380_{5}$ \\
\hline & $F_{1}$ & I 056 & I IOI $(* * *)$ & 2468 & $2539(* *)$ & $3 \times 89$ & $3294(* *)$ \\
\hline & $\mathrm{F}_{2}$ & I I I 9 & I $149\left(^{*}\right)$ & 2633 & $2795(* * *)$ & 3373 & 35 I $5\left(^{*}\right)$ \\
\hline & $\mathrm{F}_{3}$ & I I 93 & I $354\left(^{*}\right)$ & 2730 & $2858(* *)$ & $35^{8 I}$ & 3667 \\
\hline & $\mathrm{F}_{4}$ & I ooo & I $046(* * *)$ & 2579 & $2695(* * *)$ & 3064 & $3262(* * *)$ \\
\hline
\end{tabular}

(*) Différence entre $A$ et I significative au seuil o,05.

(**) Différence significative au seuil o,or.

$(* * *)$ Différence significative au seuil o,oor.

TABLEAU 2

Évolution du poids des animaux provenant de souches sélectionnées en open-field, élevés en cages Evolution of weight of animals from lines selected in open-field, in cage

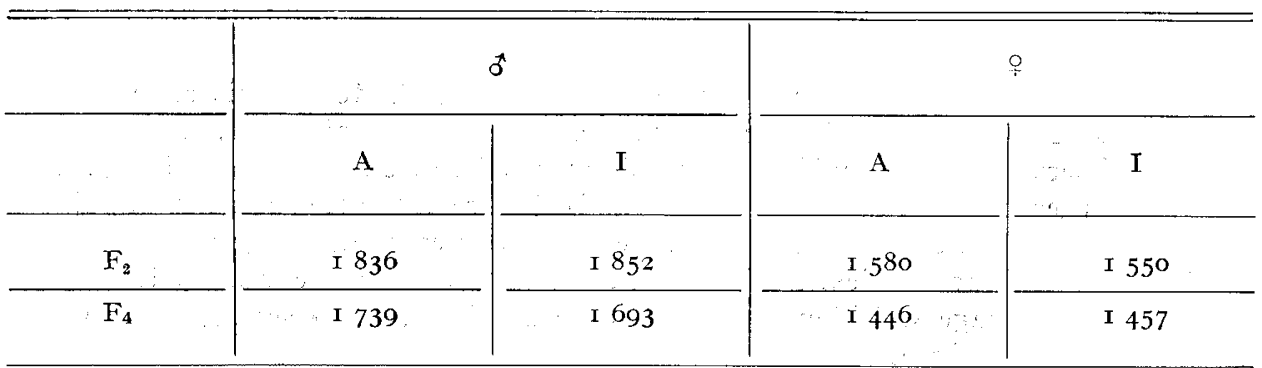

Ce poids a été mesuré à 7 semaines et non à 6 comme dans le tableau $\mathbf{I}$. 
TABLEAU 3

Evolution des caractères de conformation des souches sélectionnées en open-field Evolution of conformation characters of lines selected in open field

\begin{tabular}{|c|c|c|c|c|c|c|c|c|c|}
\hline \multirow{2}{*}{\multicolumn{2}{|c|}{$\begin{array}{l}\text { Générations } \\
\text { de sélection }\end{array}$}} & \multicolumn{2}{|c|}{$\begin{array}{l}\text { Angle poitrine } \\
\text { (grades) }\end{array}$} & \multicolumn{2}{|c|}{$\begin{array}{l}\text { Longueur } \\
\text { bréchet } \\
(\mathrm{mm})\end{array}$} & \multicolumn{2}{|c|}{$\begin{array}{l}\text { Longueur tarse } \\
\qquad(\mathrm{mm})\end{array}$} & \multicolumn{2}{|c|}{$\begin{array}{l}\text { Diamètre tarse } \\
\text { (I/10 } \mathrm{mm})\end{array}$} \\
\hline & & A & I & A & I & A & I & A & I \\
\hline \multirow{5}{*}{ ठే } & $\mathrm{F}_{0}$ & 73,9 & 74,8 & 88,3 & 89,8 & 98,7 & 99,5 & 107,8 & $\begin{array}{c}\text { I I } 0,5 \\
(* *)\end{array}$ \\
\hline & $F_{1}$ & $7 \mathrm{I}, 7$ & 72,7 & 91,8 & $9 \mathrm{I}, \mathrm{I}$ & 95.6 & 95,6 & 105,4 & 105,8 \\
\hline & $F_{2}$ & 70,8 & 71,9 & 87,6 & $88, \mathrm{I}$ & 94,7 & 95,5 & 104,7 & I 06, 3 \\
\hline & $F_{3}$ & 70,7 & $\begin{array}{c}73,5 \\
(* * *)\end{array}$ & $9 \mathrm{I}, 9$ & $\begin{array}{c}93, \mathrm{I} \\
(*)\end{array}$ & 98,6 & $\begin{array}{l}100,4 \\
(* * *)\end{array}$ & I 06,8 & $\begin{array}{l}109,9 \\
(* * *)\end{array}$ \\
\hline & $\mathrm{F}_{4}$ & 68,0 & $\begin{array}{c}69,7 \\
(* * *)\end{array}$ & 82,9 & $\begin{array}{c}84,7 \\
(*)\end{array}$ & 93,8 & $\begin{array}{c}96,0 \\
(* * *)\end{array}$ & IOI,3 & IOI, 2 \\
\hline \multirow{5}{*}{ 우우 } & $\mathrm{F}$ & 74,5 & 75,0 & 85,0 & 84,3 & 93,8 & $93, \mathbf{I}$ & 99,0 & 98,7 \\
\hline & $F_{1}$ & $\begin{array}{c}71,9 \\
(* * *)\end{array}$ & 74,5 & $8_{4}, 8$ & 85,3 & 88,3 & $\begin{array}{c}89,5 \\
(* * *)\end{array}$ & 94,9 & $\begin{array}{c}96,9 \\
(* * *)\end{array}$ \\
\hline & $F_{2}$ & 71,6 & 72,3 & 82,5 & 83,5 & 88,6 & 89,4 & 96,4 & $\begin{array}{l}97,9 \\
(*)\end{array}$ \\
\hline & $F_{3}$ & $7^{0,2}$ & $\begin{array}{l}72,8 \\
(* *)\end{array}$ & 85,7 & $\begin{array}{c}86,8 \\
(*)\end{array}$ & 90,9 & $\begin{array}{c}93, I \\
(* * *)\end{array}$ & 96,5 & $\begin{array}{l}98,6 \\
(* *)\end{array}$ \\
\hline & $\mathrm{F}_{4}$ & 67,2 & $\begin{array}{c}69,6 \\
(* * *)\end{array}$ & 78,0 & 78,8 & 87,8 & $\begin{array}{l}89,9 \\
(* * *)\end{array}$ & 93.5 & 94,4 \\
\hline
\end{tabular}

(*) Différences significatives à 0,05 .

$(* *)$ Différences significatives à o,or. génération.

genération.

tarse) et en largeur (diamètre du tarse) des os longs. Toute la croissance des animaux inactifs est donc améliorée par rapport à celle des animaux actifs et la différence observée pour le poids ne peut donc pas être due seulement à une différence dans les dépôts adipeux. De plus, il faut faire très attention à l'appellation des souches qui sont sélectionnées pour leur activité en open-field, milieu nouveau, à l'âge de deux jours; il n'est donc pas du tout impossible que leur activité dans les conditions d'élevage soit identique et c'est ce que laisse supposer une observation subjective non chiffrée.

Ces résultats sont en contradiction avec les résultats de Brown et Nestor (I973, I974 $a$ et $b$ ) montrant que les souches les plus sensibles aux stesss sont les 
plus légères. Ils peuvent cependant être conciliés si on suppose que dans nos soucshe il $\mathrm{y}$ a eu une dérive génétique entraînant une augmentation du poids des animaux de la souche inactive. En effet, dans nos conditions d'élevage très peu stressantes, cet effet pourrait se manifester alors que dans des conditions plus dures (élevage en cage) ces différences sont annulées.

L'hypothèse de la dérive génétique est vraisemblable puisque nos deux souches sont reproduites avec une structure pédigrée de Io coqs et 50 poules par souche, soit un nombre de reproducteurs faible. Afin de préciser si cette différence n'est pas due à une corrélation entre l'activité en open-field et la croissance, nous étudierons l'activité en open-field de souches sélectionnées pour la forme de leur courbe de croissance (RICARD, I975) et les corrélations génétiques et phénotypiques existant entre ces deux caractères.

$$
\text { Reçu pour publication en mai } 1977 .
$$

\section{Summary}

Relationship between growth and open-field activity in the Hen. I. - Effect of selection for open-field activity on weight growth and conformation

We have compared the growth of two strains selected for their activity in open-field (mea sure of emotivity). We find out that the most emotive birds (inactive) have a better growth for weight as well as for growth of long bones. These data are at variance with the bibliographical data which show that the animals the most sensitive to stresses or the most stressed ones have a lower growth.

This contradiction is explained in supposing that a genetical drift, due to the few number of birds, occurred.

\section{Références bibliographiques}

Brown K. I., Nestor K. E., I973. Some physiological responses of turkeys selected for high and low adrenal response to cold stress. Poultry Science, 52, I948-1954.

Brown K. I., Nestor K. E., I974a. Implications of selection for high and low adrenal response to stress. Poultry Science, 52, г 297-1306.

Brown K. I., Nestor K. E., 1974b. Effect of stress on rate of gain and feed conversion of Ohio low and high adrenal response turkey lines. Ohio Agric. Res. Develop. Center, Res. Summary, 80, 4-6.

Delpech P., Ricard F. H., I965. Relation entre les dépôts adipeux viscéraux et les lipides corporels chez lo poulet. Ann. Zootech., 14, I 8 I-I 89.

Ebedes G. A. L., I974. Effect of cage density on body weights, body weight variability and feed. intake of layer replacement pullets. $X V$ World's Poultry Congress, New Orleans, I I-r 6 August.

FaURE J. M., I975. Étude des liaisons entre comportement en open-field et émotivité chez le jeune poussin. Ann. Génét. Sél. anim., 7, I97-204.

Faure J. M., Folmer J. C., I975. Étude génétique de l'activité précoce en open-field du jeune poussin. Ann. Génét. Sél. anim., 7, г23-г 32.

Manoharan G. A., Michael R. D., I974. Some factors affecting growth in broiler chicks. Indian veterinary Journal, 51, 404-407.

Ricard F. H., 1975. Essai de sélection sur la forme de la courbe de croissance chez le poulet. Ann. Génét. Sél. anim., 7, 427-443.

Rombauts P., Faure J. M., r977. Influence de la sélection pour l'activité en open-field sur le taux de corticostérone plasmatique (à paraître).

Scholtyssek S., I973. (The influence of management system on broiler slaughter results). Archiv. Geflügelkunde, 37, 213-2 I9. 